\title{
Flegmasia cerulea dolens. Reporte de caso
}

\author{
Phlegmasia Cerulea Dolens. Case report
}

Julio Enrique Yáñez-Luque 1,a

RESUMEN

Flegmasia cerúlea dolens (flebitis, cianosis, dolor) es una enfermedad rara y con menor frecuencia de presentación en el miembro superior (5-10\%) que se caracteriza por cianosis marcada, edema y dolor intenso, causada por obstrucción del retorno venoso, es reversible si se trata a tiempo; la mitad de casos pueden progresar a gangrena del miembro con la perdida posterior del mismo. Se presenta el caso de una mujer de 48 años que ingresó por emergencia con dolor abdominal intenso y posteriormente presentó trastorno del sensorio y cianosis marcada de mano derecha. Se hizo el diagnóstico de intoxicación por metanol y flegmasia cerúlea dolens, apoyados en los resultados de la ecografía Doppler y el angioTEM. Se realizó fasciotomía e inició enoxaparina con la posterior recuperación, alta y control por consultorio.

PALABRAS CLAVE: Tromboflebitis, trombosis de la vena, amputación. (Fuente: DeCS BIREME).

\section{SUMMARY}

Phlegmasia cerulea dolens (phlebitis, cyanosis, pain) is a rare disease that uncommonly affects the upper limbs (5$10 \%$ ). It is characterized by marked cyanosis, edema and severe pain caused by obstruction of the vein flow. The condition is reversible if treated on time; half of the cases may progress to limb gangrene with potential for losing the extremity. We present the case of a 48 -year-old female patient who was admitted to the emergency room with severe abdominal pain, altered sensory status and marked cyanosis of the right hand. The diagnosis was methanol intoxication and phlegmasia cerulea dolens based on the findings of a Doppler ultrasound and angio CT scan. Fasciotomy was performed and enoxaparin was started with full recovery.

KEYWORDS: Thrombophlebitis, venous thrombosis, amputation. (Source: MeSH NLM).

\section{INTRODUCCIÓN}

Flegmasia cerúlea dolens (FCD) (La Phlebite bleue) es un cuadro severo de trombosis venosa profunda caracterizada por obstrucción venosa, edema marcado, cianosis y dolor, acompañado de espasmo arterial marcado, que puede llegar a la gangrena venosa. En 1593 Fabricius Hildanus fue el primero en postular la posibilidad de gangrena venosa (1), y en 1938 Gregoire utiliza el término flegmasia cerúlea dolens para diferenciarla de la flegmasia alba dolens en la que no hay isquemia (2).
FCD es reversible pero puede llevar a la gangrena y pérdida posterior de la extremidad. Esta entidad tiene una mortalidad de alrededor de $25 \%$ y una tasa de amputación entre 12 y $25 \%$ (3), por lo cual es una entidad a tener en cuenta en pacientes graves.

\section{PRESENTACIÓN DEL CASO}

Mujer de 48 años de edad con antecedentes médicos no significativos con historia de ingesta de alcohol tres veces por semana, acudió al servicio de emergencia por presentar dolor abdominal intenso, náuseas y

Servicio de Cuidados Críticos, Hospital Santa Rosa. Lima, Perú.

a Médico Intensivista 
vómitos de tres horas de evolución. Al examen físico se encontró PA: 200/100 mm Hg, FC: 105x', FR: 25, T: $37^{\circ} \mathrm{C}$, pulsos presentes, estaba despierta y refería dolor muy intenso en abdomen. Los resultados de los exámenes de laboratorio al ingreso se muestran en la tabla 1. Una hora después del ingreso aumentó el

Tabla 1. Exámenes de laboratorio al ingreso.

\begin{tabular}{ll}
\hline Laboratorio & Valor \\
Glucosa & $388 \mathrm{mg} / \mathrm{dl}$ \\
Tiempo de Protrombina & $11 \mathrm{seg}$ \\
Tiempo Tromboplastina & $30 \mathrm{seg}$ \\
Parcial & 22900 leucocitos, $4 \%$ \\
Hemograma & abastonados \\
Hemoglobina & $7,5 \mathrm{~g} / \mathrm{dl}$ \\
Recuento de Plaquetas & $374000 \mathrm{x} \mathrm{mm}{ }^{3}$ \\
Creatinina & $1,05 \mathrm{mg} / \mathrm{dl}$ \\
Sodio & $143 \mathrm{mEq} / 1$ \\
Potasio & $5,2 \mathrm{mEq} / 1$ \\
Gases arteriales $^{\text {pH }}$ & \\
PO $_{2}$ & 6,8 \\
$\mathrm{PCO}_{2}$ & $200 \mathrm{~mm} \mathrm{Hg}$ \\
$\mathrm{HCO}_{3}$ & $29 \mathrm{~mm} \mathrm{Hg}$ \\
\hline
\end{tabular}

dolor y presentó trastorno del sensorio, requiriendo de intubación endotraqueal y ventilación mecánica, se utilizó bicarbonato de sodio y noradrenalina con fluido terapia enérgica por acidosis metabólica severa.

Tres horas después del ingreso presentó cianosis en mano derecha con aumento de volumen. La paciente fue admitida en cuidados intensivos con diagnostico presuntivo de intoxicación por metanol a descartar trombosis arterial de mano derecha. Se corrigió el problema metabólico y luego se realizó Doppler arterial de miembro superior derecho cuyo resultado fue: "Arteria axilar, humeral, radial y cubital con presencia de flujo, ausencia de flujo en arterias interdigitales" por lo que se realizó angioTEM, el que mostró ausencia de flujo arterial interdigital y de arco palmar (Figura 1).

El edema de mano causó un síndrome compartimental (Figura 2A) y, se procedió a realizar fasciotomía de emergencia (Figura 2B) y se inició enoxaparina. Se recuperó la homeostasis de medio interno y continuó con curaciones diarias de mano derecha y elevación del miembro; progresivamente fue recuperando la coloración normal y disminuyendo el edema (Figura 2C). A los cinco días del ingresó se le retiró el tubo endotraqueal y fue dada de alta de la Unidad de cuidados intensivos a los 15 días. Dos meses después había recuperado la coloración y parcialmente la motilidad de la mano derecha (Figura 2D).
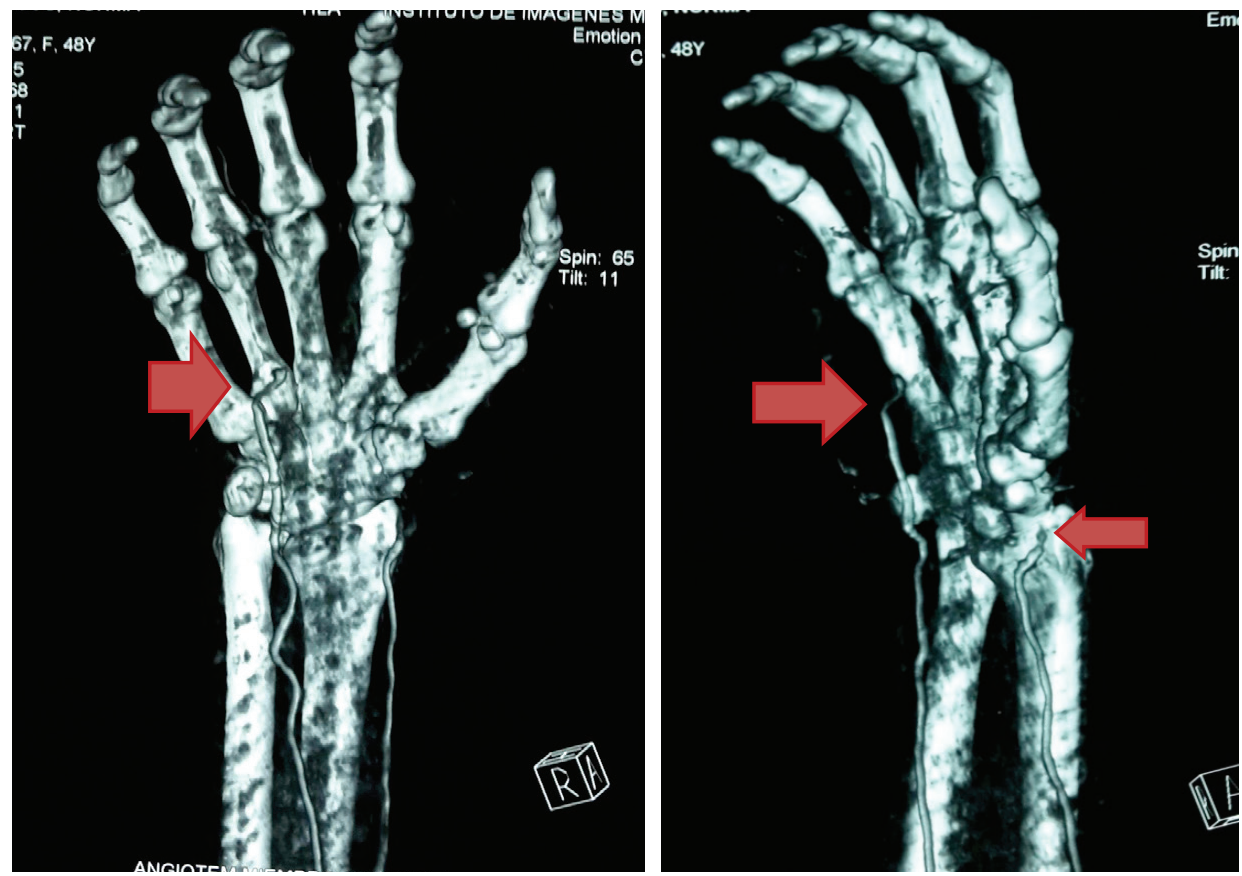

Figura 1. AngioTEM de mano derecha. Se muestra un "stop" en arterias radial y cubital y ausencia de arco palmar. 

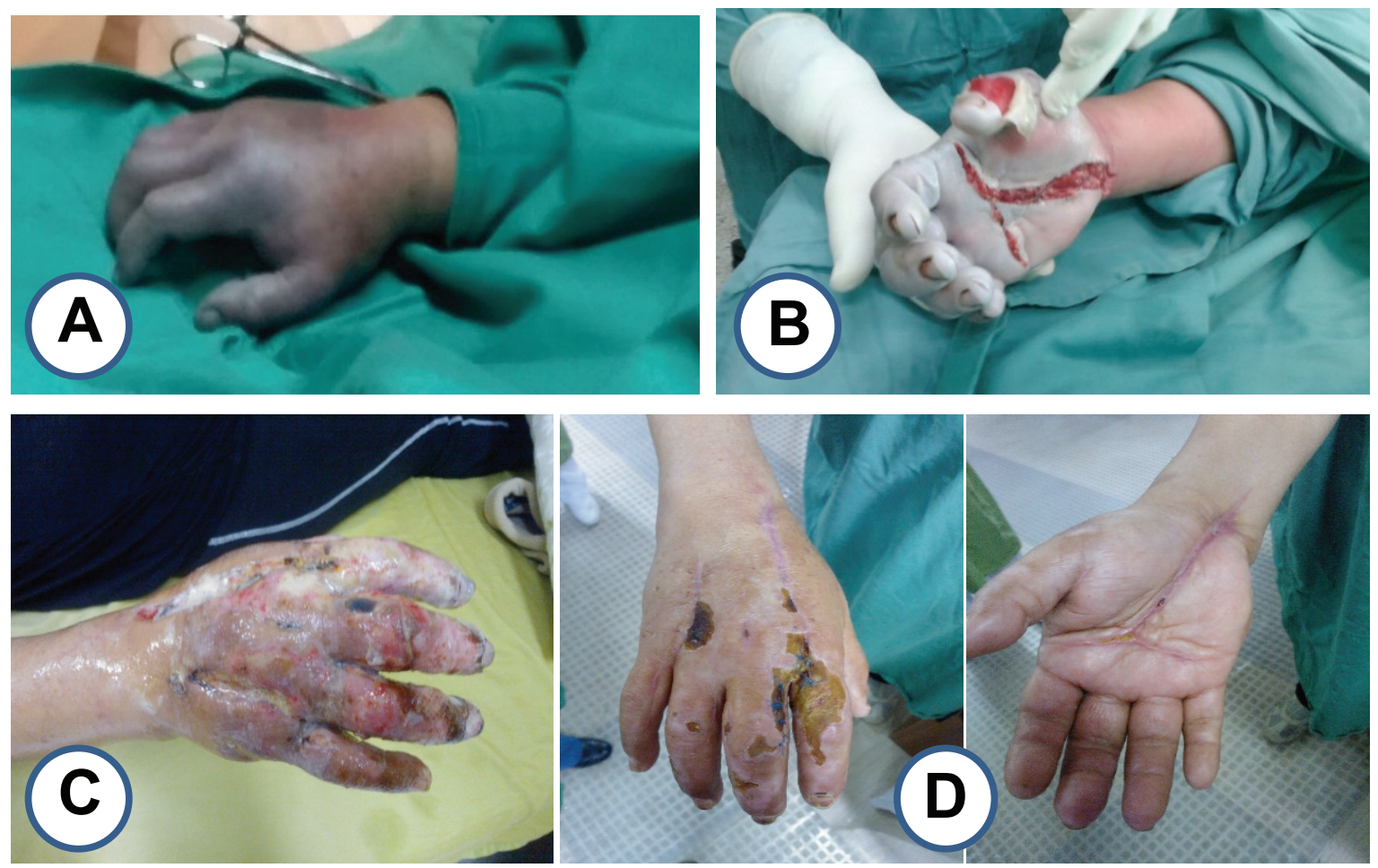

Figura 2. A: Inicio del cuadro: Cianosis y edema marcados. B: Fasciotomía: Incisión quirúrgica y disminución del edema. C: Mejoría parcial: Recuperación parcial de epidermis y ausencia de cianosis. D: Control después del alta, muestra recuperación casi total y mejoría clínica.

\section{DISCUSIÓN}

El inicio tórpido del cuadro sugería una trombosis arterial pero llamaba mucho la atención la presencia de pulso en arteria radial derecha. Debido a que la paciente cursaba con acidosis metabólica severa y shock, también se pensó en la posibilidad de un evento adverso por el uso de drogas vaso activas.

La FCD es un cuadro que usualmente se presenta en miembros inferiores y raramente se da en miembros superiores (4); es un estadio previo a la gangrena venosa de miembro la cual puede ser modificada o agravada por factores clínicos como uso de warfarina, hipotensión, uso de medicamentos vaso activos y trombosis venosa profunda (5).

La fisiopatología puede explicarse mediante los experimentos de Alton Ochsner, quien demostró que al ligar la vena principal de la pierna posterior en perros resultaba en una disminución a la mitad del volumen de pulsación en la parte distal y un aumento en la presión media venosa de 7,7 a $97 \mathrm{~cm}$. de $\mathrm{H}_{2} \mathrm{O}$ (6). Una irritación química en el lumen de la vena también producía una reducción marcada de volumen, efecto que se resolvía al interrumpir la vía nerviosa con anestésico o resección de la cadena simpática, lo que demuestra un impulso vasoconstrictor iniciado localmente a través del ganglio simpático hasta los vasos arteriales terminales (6).

Estudios histopatológicos muestran infiltración inflamatoria difusa en la capa adventicia venosa la cual puede propagarse y extenderse a la misma capa arterial, lo cual explicaría el vaso espasmo arterial severo (7); el edema marcado debido a todo lo anterior complica más la irrigación sanguínea debido a un síndrome compartimental, el cual en nuestro caso, fue tratado con fasciotomía. En el caso presentado se realizó una arteriografía la cual no mostraba vasos arteriales en la mano, pero la recuperación total demostró que el árbol arterial estaba indemne. La causa desencadenante en este caso pudo haber sido una trombosis venosa o una flebitis química debido al tratamiento de urgencia, dando como resultado el cuadro de FCD sin llegar a la gangrena del miembro superior derecho. El diagnóstico se realiza por imágenes siendo de preferencia el ultrasonido dúplex, venografía, angioTEM o resonancia magnética con venografía (8). 
El manejo inicial es reposo absoluto, elevación del miembro, resucitación con fluidos y administración de heparina, con monitoreo de plaquetas, también puede usarse heparina de bajo peso molecular. Angioplastia, angioplastia con stent, trombolisis dirigida con catéter, fasciotomía, trombectomía y bypass venoso son los tratamientos más invasivos $(8,9)$.

En resumen, la Flegmasia cerúlea dolens es un cuadro grave y raro que es un paso previo a la gangrena isquémica; el diagnostico depende del cuadro clínico y estudios de imágenes en los que no hay lesión del árbol arterial. Debe tenerse en cuenta entre los diagnósticos de isquemia con pulsos y de recibir el tratamiento adecuado es reversible.

\section{Declaración de financiamiento y de conflictos de interés:}

El reporte fue financiado por el autor, quien declara no tener conflictos de intereses.

\section{Correspondencia:}

Julio Enrique Yáñez-Luque

Calle Caracas 2377 dpto. 4 Jesús María. Lima, Perú.

Correo electrónico: Julio.yanez.1@upch.pe

\section{REFERENCIAS BIBLIOGRÁFICAS}

1. Jackson E. Phlegmasia Cerulea Dolens. American Journal of Surgery. 1958; 95(3):429-433.

2. Veltchev L, Kalniev M, Todorov T. Phlegmasia cerulea dolence - risk factors and prevention: Case report. J IMAB Annu Proc. 2009; 1: 89-91.

3. Abayasekara D, Kirk J. Phlegmasia caerulea dolens secondary to pelvic plasmacytoma and left femoral deep vein thrombosis. Int J Surg Case Rep. 2013; 4(10): 825-827.

4. Standeven A. Venous gangrene. Postgrad Med J. 1958; 34(395): 470-47.

5. Warkentin $\mathrm{T}$. Ischemic limb gangrene with pulses. $\mathrm{N}$ Engl J Med. 2015; 373:642-55.

6. De Bakey M, Burch G, Ochsner A. Effect of chemical irritation of a venous segment on peripheral pulse volume. Proc Soc Exp Biol. 1939; 41: 585-590.

7. Haimovici H. Gangrene of the extremities of venous origin review of the literature with case reports. Circulation. 1950; 1:225-240.

8. Chinsakchai K, Duis K, Moll F, de Borst G. Trends in management of Phlegmasia Cerulea Dolens. Vasc Endovascular Surg. 2011; 45(1):5-14.

9. Klok FA, Huisman MV. Seeking optimal treatment for phlegmasia cerulea dolens, Thromb Res. 2013; 131(4):372-3.

Recibido: 03/03/2016

Aceptado: 20/06/2016 